\title{
Láminas delgadas ferroeléctricas de titanato de plomo modificado con un $20 \%$ de lantano
}

\author{
M. ALGUERÓ, M.L. CALZADA Y L. PARDO \\ Instituto de Ciencia de Materiales, CSIC. Madrid 28049. España
}

\begin{abstract}
Se han preparado láminas delgadas ferroeléctricas de titanato de $\mathrm{Pb}$ modificado con un $20 \%$ de $\mathrm{La}, \mathrm{Pb}_{0.7} \mathrm{La}_{0.2} \mathrm{TiO}_{3^{\prime}}$ por una técnica sol-gel basada en la ruta de los dioles. Se ha variado el contenido inicial de PbO, del nominal a un exceso del $20 \%$ molar; y la velocidad de calentamiento en el tratamiento térmico de cristalización a $650^{\circ} \mathrm{C}$, de $10^{\circ} \mathrm{C} \mathrm{min}^{-1}$ a más de $500^{\circ} \mathrm{C} \mathrm{min}{ }^{-1}$, y estudiado su efecto en la microestructura, estructura y composición de las láminas. No es posible obtener láminas monofásicas con el tratamiento con $10^{\circ} \mathrm{C} \mathrm{min}^{-1}$, aun compensando la volatilización de $\mathrm{PbO}$ con un exceso inicial del $20 \%$ molar. Sí lo es con tratamiento rápido manteniendo dicho exceso de $\mathrm{PbO}$. Estas últimas láminas presentan una permitividad dieléctrica a temperatura ambiente de $\varepsilon=700$, y una variación entre ambiente y $100^{\circ} \mathrm{C}$ de $\Delta \varepsilon=75$, parámetros muy interesantes para memorias DRAMs.
\end{abstract}

Palabras clave: láminas ferroeléctricas, $\mathrm{Pb}_{0.7} \mathrm{La}_{0.2} \mathrm{TiO}_{3}$, memorias DRAM

Lead titanate ferroelectric thin films modified with $20 \%$ lanthanum

Lanthanum modified lead titanate ferroelectric thin films with a composition $\mathrm{Pb}_{0.7} \mathrm{La}_{0.2} \mathrm{TiO}_{3}$ have been prepared by a diol based sol-gel technique. The effect on the film structure, microstructure and composition of the initial PbO content, from the nominal value to an excess of $20 \mathrm{~mole} \%$, and of the heating rate of the thermal treatment of crystallisation at $650^{\circ} \mathrm{C}$, from $10^{\circ} \mathrm{C}$ $\mathrm{min}^{-1}$ to more than $500^{\circ} \mathrm{C} \mathrm{m_{n } ^ { - 1 } \text { , has been studied. It is not possible to obtain single phase films with the treatment at } 1 0 ^ { \circ } \mathrm { C } \text { min }}$ ${ }_{1}^{1}$, even when $\mathrm{PbO}$ volatilisation is compensated with an initial excess of $\mathrm{PbO}$ of $20 \mathrm{~mole} \%$. It is possible with the treatment at more than $500^{\circ} \mathrm{C} \mathrm{min}-1$ and the latter excess of $\mathrm{PbO}$. These films present a relative dielectric permittivity at room temperature of $\varepsilon=700$, and a variation between room temperature and $100^{\circ} \mathrm{C}$ of $\Delta \varepsilon=75$, being both parameters very interesting for DRAMs.

Key words: ferroelectric films, $\mathrm{Pb}_{0.7} \mathrm{La}_{0.2} \mathrm{TiO}_{3}$, DRAMs

\section{INTRODUCCIÓN}

En las memorias dinámicas de acceso aleatorio (DRAMs) el elemento activo es un condensador, y los dos estados de la memoria se definen como los estados cargado y descargado del condensador. La demanda de memorias de capacidad creciente manteniendo, o incluso reduciendo, las dimensiones, exige el uso de materiales de permitividad dieléctrica creciente. Los óxidos ferroeléctricos, con permitividades dos órdenes de magnitud mayores que los compuestos $\mathrm{SiO}_{2} / \mathrm{Si}_{3} \mathrm{~N}_{4}$ actualmente utilizados, son los principales candidatos (1). Las composiciones idóneas son aquellas que presentan la transición ferro-paraeléctrica a temperatura ambiente, ya que la anomalía dieléctrica lleva asociada valores altos de la permitividad, y desorden en la ocupación de uno de los sitios de la estructura, lo que se traduce en una transición difusa y, por tanto, en que los valores de permitividad altos se mantengan en un intervalo de temperaturas apreciable. El material más investigado es el titanato de bario modificado con estroncio (BST), alrededor de la composición $\mathrm{Ba}_{0.7} \mathrm{Sr}_{0.3} \mathrm{TiO}_{3}$ (2). Sin embargo, existen otros candidatos. La temperatura de la transición ferro-paraeléctrica en el titanato de plomo modificado con La (PTL) disminuye al aumentar la cantidad de La (3). La composición con un $28 \%$ es paraeléctrica a temperatura ambiente.

Esta comunicación recoge la ampliación de nuestro trabajo en láminas delgadas ferroeléctricas de titanato de $\mathrm{Pb}$ modificado con un $8 \%$ de La $(4,5)$ a un $20 \%$ de La. Se discuten los problemas que aparecen en la preparación al aumentar la cantidad de modificador, y su potencial para DRAMs.

\section{MÉTODOS EXPERIMENTALES}

Las soluciones precursoras de titanato de $\mathrm{Pb}$ modificado con un $20 \%$ de $\mathrm{La}, \mathrm{Pb}_{0.7} \mathrm{La}_{0.2} \mathrm{TiO}_{3}$, (PTL20) se sintetizaron por una técnica sol-gel que consta de dos etapas: la síntesis de un sol de $\mathrm{Pb}$ y Ti por la llamada ruta de los dioles (6), y la adición posterior del modificador en forma de sal disuelta en agua (7). Es básicamente el mismo procedimiento que se empleó con éxito en la preparación de láminas de PTL con un $8 \%$ de La (PTL8), cuyos detalles se pueden encontrar en $(4,5)$. El uso de cantidades mayores de acetato de La hace necesaria la adición en la segunda etapa de ácido acético hasta reducir el pH a 4.5 con el fin de lograr la disolución de la sal. Se prepararon soluciones con tres composiciones; la nominal, $\mathrm{Pb}_{0.7} \mathrm{La}_{0.2} \mathrm{TiO}_{3}$, y con excesos de $\mathrm{PbO}$ del 10 y del $20 \%$ molar. Las soluciones se depositaron sobre sustratos de $\mathrm{Pt} / \mathrm{TiO}_{2} / \mathrm{Si}$ por goteo y centrifugación. Los depósitos húmedos así obtenidos se calentaron en una placa calefactora a $350^{\circ} \mathrm{C}$ durante 1 minuto para su secado. Esta etapa del proceso se repitió tres veces con el fin de alcanzar espesores mayores. Finalmente, los depósitos secos se calentaron en un horno a $650^{\circ} \mathrm{C}$ durante 12 minutos para su cristalización. Se emplearon dos velocidades de calentamiento, $10^{\circ} \mathrm{C} \min ^{-1} \mathrm{o}$ más de $500^{\circ} \mathrm{C} \mathrm{min}{ }^{-1}$. La velocidad más alta se consigue mediante la introducción de la lámina en el horno precalentado, procedimiento al que nos referiremos como calentamiento rápido a partir de ahora.

El espesor de las láminas se midió con un perfilómetro (Form Talysurf-50 Series 2 de Taylor Hobson). La distorsión tetragonal de la estructura perovskita y la posible presencia de 
segundas fases se estudiaron por difracción de rayos $X$ con ángulo rasante (Siemens D-500, radiación $\mathrm{Cu} \mathrm{K}_{\alpha}$ ). La microestructura se estudió por microscopía electrónica de barrido (Hitachi S 800). La relación atómica $\mathrm{Pb} / \mathrm{Ti}$ se midió en el mismo microscopio por espectroscopía de rayos $\mathrm{X}$ por dispersión de energía (Quantum Kevex). Detalles de las medidas se pueden encontrar en (5). La caracterización eléctrica de las láminas se realizó tras el depósito sobre su superficie de electrodos de Pt de $500 \mu \mathrm{m}$ de diámetro. En primer lugar se midieron ciclos de densidad de corriente a.c. (amplificador de corriente Keithley 428) y ciclos de histéresis ferroeléctricos (circuito Sawyer-Tower). Sin embargo, los ciclos estaban fuertemente deformados por contribuciones de conducción. La polarización conmutable se evaluó mediante la integración de la corriente de conmutación de la polarización en experimentos con una secuencia de tres pulsos, en los que se pueden sustraer la carga de conducción. Detalles de estas medidas se pueden encontrar también en (5). A continuación, se midió la dependencia de la pemitividad dieléctrica entre temperatura ambiente y $500^{\circ} \mathrm{C}$ (analizador de impedancias HP 4284A). En

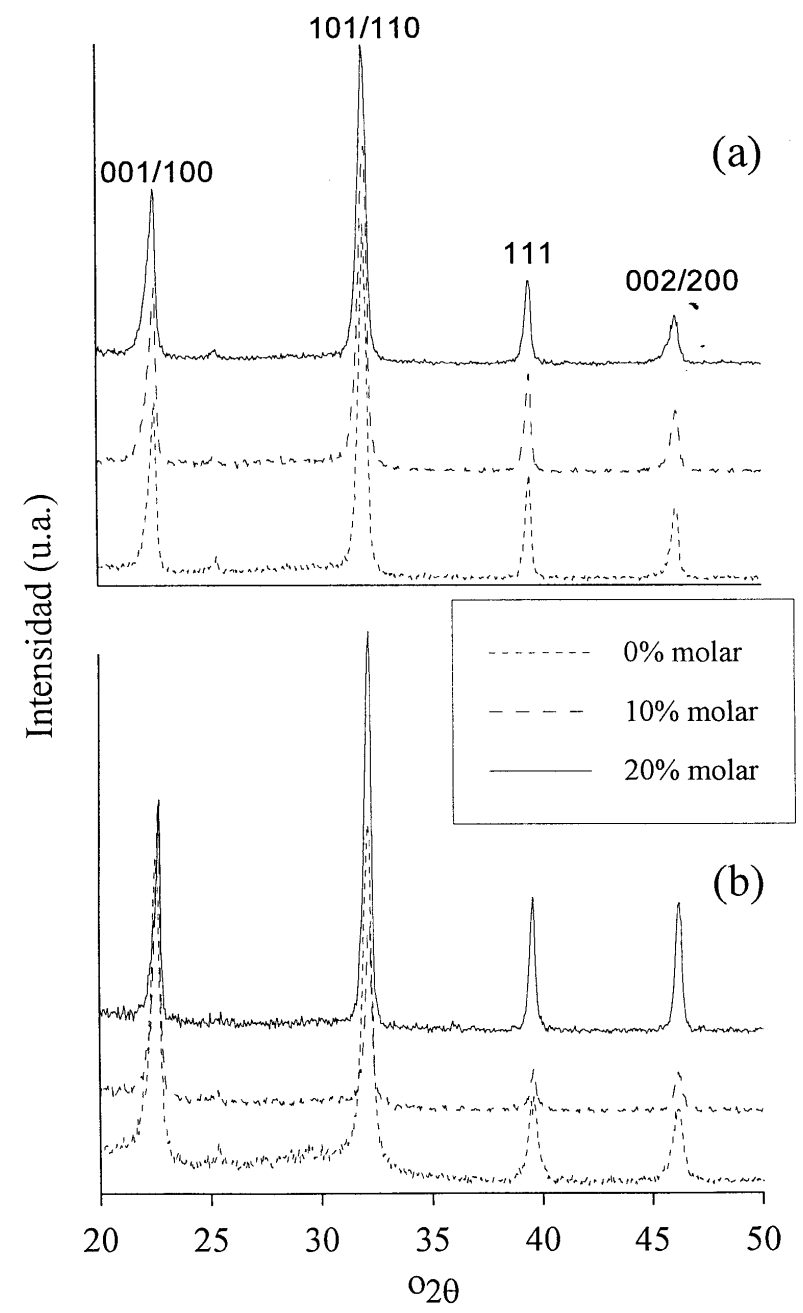

Figura 1. Diagramas de difracción de rayos $\mathrm{X}$ con ángulo rasante (20) de las láminas de PTL20 preparadas con distintos contenidos iniciales de $\mathrm{PbO}$, y tratadas (a) con $10^{\circ} \mathrm{C} \mathrm{min}^{-1}$, (b) con calentamiento rápido. estas medidas se calentó la muestra a $500^{\circ} \mathrm{C}$ y se midió durante el enfriamiento.

\section{RESULTADOS}

Los espesores de las distintas láminas preparadas se dan en la Tabla I. No se observa ninguna dependencia con el contenido inicial de $\mathrm{PbO}$ o la velocidad de calentamiento, siendo el valor medio de $365 \mathrm{~nm}$.

Los diagramas de difracción de rayos $\mathrm{X}$ se muestran en la Figura 1. La estructura perovskita, cuyos picos están etiquetados en la figura con sus índices de Miller, es la única presente en las láminas tratadas con $10^{\circ} \mathrm{C} \min ^{-1}$ (Fig.1(a)). El pico de poca intensidad en $\sim 25^{\circ} 2 \theta$ corresponde a la capa de $\mathrm{TiO}_{2}$ del sustrato. En cambio, en el difractograma de la lámina tratada por calentamiento rápido (Fig.1(b)), y preparada a partir de la solución con la composición nominal, aparece un pico muy ancho alrededor de $30^{\circ} 2 \theta$ que podría estar relacionado con una segunda fase pobremente cristalizada. Este pico no aparece en
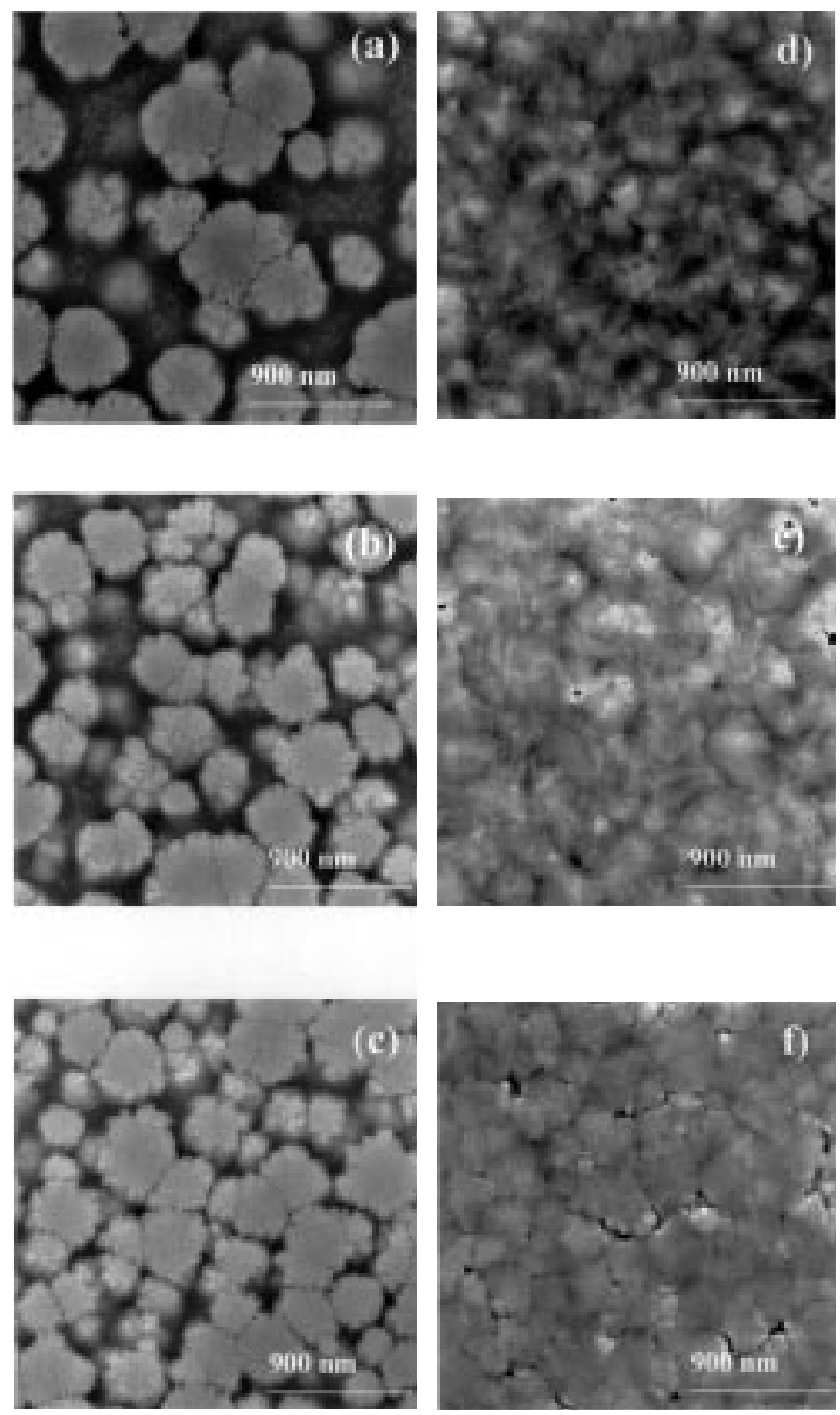

Figura 2. Micrografías de las láminas de PTL20 preparadas con un contenido inicial de PbO del (a, d) 0\% molar, (b, e) 10\% molar, y (c, f) $20 \%$ molar, tratadas con $(\mathrm{a}-\mathrm{c}) 10^{\circ} \mathrm{C} \mathrm{min}^{-1} \mathrm{y}(\mathrm{d}-\mathrm{f})$ calentamiento rápido. 
los difractogramas de láminas preparadas a partir de soluciones con excesos de $\mathrm{PbO}$. La perovskita no presenta textura apreciable en ninguno de los casos. Su distorsión tetragonal en las distintas láminas se da en la Tabla I. No muestra tendencias claras con la composición inicial, pero si es mayor en las tratadas con $10^{\circ} \mathrm{C} \mathrm{min}^{-1}, \sim 1.008$, que en las tratadas con calentamiento rápido, $\sim 1.005$.

Las micrografías de las láminas se muestran en la Figura 2. Aquellas tratadas con $10^{\circ} \mathrm{C} \mathrm{min}{ }^{-1}$ muestran una microestructura heterogénea. Comprende agrupaciones de granos con un tamaño de entre 70 y $100 \mathrm{~nm}$ en distintos estadios de sinterización, y una segunda fase menos emisora muy porosa. El porcentaje de superficie constituido por las agrupaciones de granos crece con el exceso inicial de $\mathrm{PbO}$. Medidas de EDXS dirigiendo el haz de electrones sobre los dos tipos microestructurales muestran que la relación atómica $\mathrm{Pb} / \mathrm{Ti}$ es mayor en las agrupaciones de granos, entre 0.77 y 0.81 , que en la segunda fase menos emisora, 0.3. Medidas barriendo el haz en áreas de $100 \mu \mathrm{m}^{2}$ muestran que la relación media aumenta con el contenido inicial de $\mathrm{PbO}$ alcanzando el valor nominal, 0.70 , en la preparada con un $20 \%$ de exceso. La microestructura de las láminas tratadas por calentamiento rápido está formada por granos con un tamaño de entre 100 y 500 nm, cuyos bordes se definen progresivamente al aumentar el contenido inicial de $\mathrm{PbO}$. El valor medio de la relación $\mathrm{Pb} / \mathrm{Ti}$ en estas últimas láminas aumenta igualmente con el contenido inicial

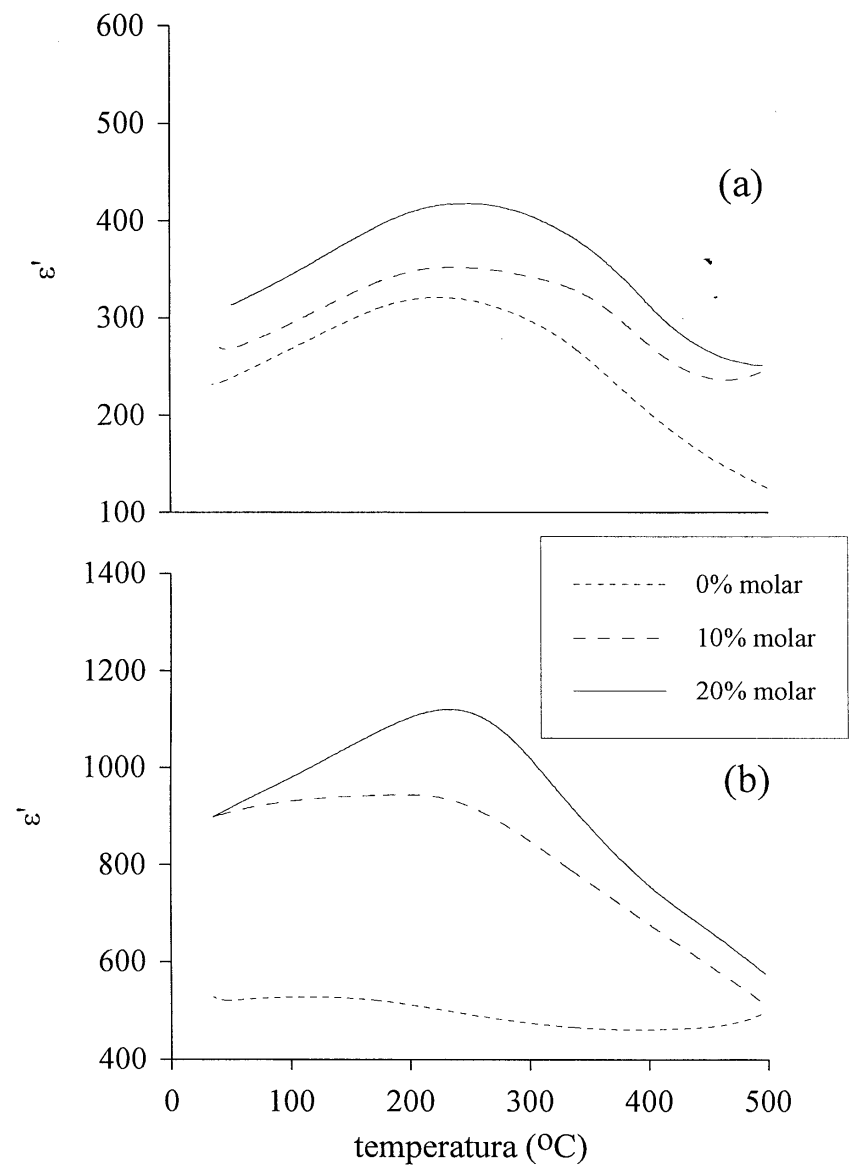

Figura 3. Dependencia de la permitividad dieléctrica con la temperatura de las láminas de PTL20 preparadas con distintos contenidos iniciales de $\mathrm{PbO}, \mathrm{y}$ tratadas (a) con $10^{\circ} \mathrm{C}$ min-1, (b) con calentamiento rápido. de $\mathrm{PbO}$, alcanzándose el valor de 0.7 en la del $20 \%$ molar. Los resultados para las 6 láminas se dan en la Tabla I.

Los experimentos de conmutación de la polarización pusieron de manifiesto el carácter ferroeléctrico de las láminas, pero también la presencia de fenómenos de conducción significativos. Los valores de polarización conmutada, $\mathrm{P}_{\mathrm{sw}^{\prime}}$ con $300 \mathrm{kV}$ $\mathrm{cm}^{-1}$ se dan en la Tabla I. La dependencia de la permitividad dieléctrica con la temperatura se muestra en la Figura 3. En todas las láminas, excepto en la tratada por calentamiento rápido sin exceso de $\mathrm{PbO}$ inicial, se observa un máximo muy ancho correspondiente a la transición ferro-paraeléctrica. Los valores de la permitividad dieléctrica y la tangente de pérdidas a temperatura ambiente antes del experimento, asi como la temperatura de la transición, se dan en la Tabla I.

\section{DISCUSIÓN}

Aunque los resultados de difracción de rayos $\mathrm{X}$ en las láminas tratadas con $10^{\circ} \mathrm{C} \mathrm{min}{ }^{-1}$ parecen indicar que no contienen segundas fases, la microscopía electrónica combinada con EDXS pone de manifiesto que sí existe una fase muy deficiente en $\mathrm{Pb}$. Una fase similar ya se había observado en las láminas con un $8 \%$ de La preparadas sin exceso de $\mathrm{PbO}$ inicial (5). Sin embargo, mientras que en estas últimas la segunda fase desaparecía cuando se añadía un $10 \%$ molar de exceso de $\mathrm{PbO}$, en las láminas con un $20 \%$ de La un $20 \%$ molar de exceso no es suficiente para su desaparición. Nótese que está última lámina contiene segunda fase a pesar de presentar una relación atómica media $\mathrm{Pb} / \mathrm{Ti}$ igual a la nominal. La relación atómica de las agrupaciones de granos de perovskita es de $\sim 0.78$. Considerando que el La sustituye al $\mathrm{Pb}$ en las posiciones A de la estructura, dicho valor de la relación $\mathrm{Pb} / \mathrm{Ti}$ corresponde a una relación $\mathrm{La} / \mathrm{Ti}$ de $\sim 0.15$. Por otro lado, los valores de la temperatura de transición obtenidos en estas láminas, $\sim 239^{\circ} \mathrm{C}$, son significativamente mayores que los que presentan materiales cerámicos con la misma composición nominal, $180^{\circ} \mathrm{C}$ (3). Como ya se dijo, la temperatura de la transición disminuye en el sistema PTL al aumentar el contenido de La en solución sólida (3). Entonces, el mayor valor de la temperatura en las láminas indica también que no todo el La se incorpora en la estructura. Estos resultados parecen indicar que en algún momento del tratamiento térmico de cristalización con $10^{\circ} \mathrm{C} \mathrm{min}{ }^{-1}$ se produce una separación de fases que impide la formación de una

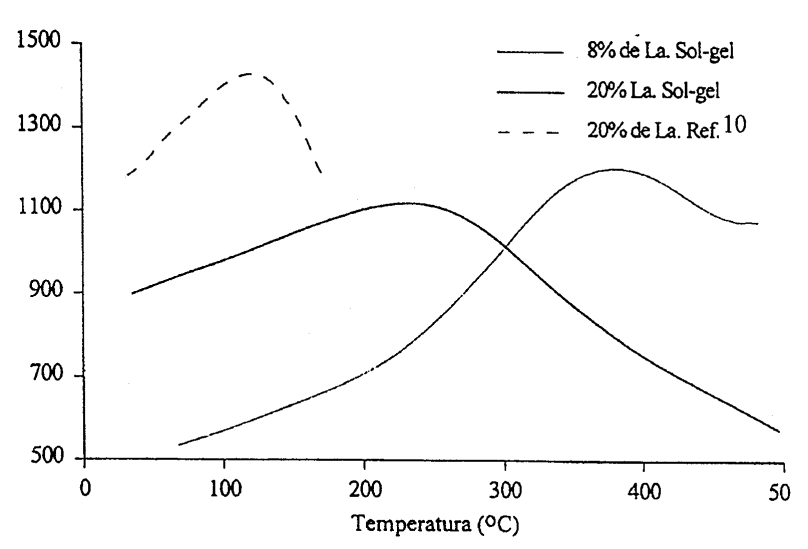

Figura 4. Comparación de las dependencias con la temperatura de la permitividad dieléctrica de distintas láminas de PTL. 
lámina homogénea. Fenómenos de este tipo se han observado en zirconato titanato de plomo (PZT) (8).

Los resultados de difracción de rayos $\mathrm{X}$ indican que sí existe una segunda fase pobremente cristalizada en las láminas sin exceso de $\mathrm{PbO}$ inicial tratadas por calentamiento rápido. Una fase similar en láminas con un $8 \%$ de La resultó ser una fase intermedia en transformación en la estructura perovskita, que se estabilizaba por deficiencia de $\mathrm{Pb}$ (9). Excesos de $\mathrm{PbO}$ aceleraban la transformación. En las láminas con un $20 \%$ de La, la relación $\mathrm{Pb} / \mathrm{Ti}$ aumenta con el contenido inicial de $\mathrm{PbO}$ hasta el valor nominal con un exceso del $20 \%$ molar, lo que podría deberse a la presencia de cantidades decrecientes de segunda fase. Esto quiere decir que las láminas preparadas con un exceso del $20 \%$ son probablemente monofásicas, siempre que no se hayan producido fenómenos de separación de fase como los observados con $10^{\circ} \mathrm{C} \mathrm{min}{ }^{-1}$ durante el tratamiento. Sus microestructuras parecen indicar que no ocurren. Estas láminas presentan propiedades interesantes para DRAMs, a pesar de sus pérdidas dieléctricas altas. En la Figura 4 se muestra la dependencia de su permitividad dieléctrica con la temperatura, comparada con la dependencia de láminas análogas con un $8 \%$ de La, y con la de láminas con un $20 \%$ de La preparadas por pulverización catódica que en un estudio reciente (10) se han considerado adecuadas para su uso en DRAMs. Se observa que el aumento de la cantidad de La en las láminas sol-gel desplaza la transición hacia ambiente y aumenta su carácter difuso. Por lo tanto, aumentan los valores de permitividad a temperatura ambiente y disminuye la pendiente con la temperatura. En relación con la lámina estudiada en (10), la permitividad es menor pero, en cambio, la pendiente es también menor. En la lámina sol-gel la permitividad aumenta en $\Delta \varepsilon=75$ entre ambiente y $100^{\circ} \mathrm{C}$ mientras que en la lámina por pulverización catódica aumenta en 200 en el mismo intervalo de temperatura.

\section{CONCLUSIONES}

Es posible preparar láminas delgadas ferroeléctricas de titanato de $\mathrm{Pb}$ modificado con un $20 \%$ de La por una técnica solgel. Tratamientos térmicos de cristalización a $650^{\circ} \mathrm{C}$ con una velocidad de calentamiento de $10^{\circ} \mathrm{C} \mathrm{min}^{-1}$ no permiten la obtención de láminas monofásicas, ni siquiera con un exceso de $\mathrm{PbO}$ inicial del 20\% molar. La incorporación del La a la estructura perovskita es incompleta. Tratamientos térmicos de cristalización a la misma temperatura con calentamiento rápi- do sí permiten preparar láminas monofásicas con la relación $\mathrm{Pb} / \mathrm{Ti}$ diseñada, cuando se emplea un exceso inicial de $\mathrm{PbO}$ del $20 \%$ molar. Presentan valores a temperatura ambiente de la permitividad dieléctrica de 700 , de la tangente de pérdidas de 0.12 , y de polarización conmutable de $4.4 \mu \mathrm{C} \mathrm{cm}^{-2}$. Presentan una temperatura de la transición de $230^{\circ} \mathrm{C}$, y una variación de la permitividad de 75 entre temperatura ambiente y $100^{\circ} \mathrm{C}$, lo que les hace especialmente interesantes para DRAMs.

\section{AGRADECIMIENTOS}

Este trabajo se realizó gracias a la financiación de la Comisión Europea a través del proyecto Copernicus Ref. ERBCIPACT940236. Los autores agradecen al Instituto de Microelectrónica de Madrid (CNM-CSIC) las facilidades dadas para usar su microscopio electrónico de barrido.

\section{BIBLIOGRAFÍA}

1. R.E. Jones, P. Zurcher, P. Chu, D.J. Taylor, Y.T. Lii, B. Jiang, P.D. Maniar y S.J Gillispie. "Memory applications based on ferroelectric and high-permittivity dielectric thin films". Microelectronic Engineering 29, 3-10 (1995).

2. A.I. Kingon, S.K. Streiffer, C.C. Basceri y S.R. Summerfelt. "High permittivity perovskite thin films for dynamic random-access memories". MRS Bull. 21:7, 46-52 (1996).

3. T. Yamamoto, H. Igarashi y K. Okazaki. “Dielectric, electromechanical, optical and mechanical properties of lanthanum modified lead titanate ceramics". J. Am. Ceram. Soc. 66:5 363-366 (1983).

4. M.L. Calzada, M. Algueró y L. Pardo. "Chemistry crystallization microstructure relations of sol-gel derived lanthanum modified lead titanate thin films". J. Sol-Gel Sci. Tech. 13, 837-841 (1998).

5. M. Algueró, M.L. Calzada, C. Quintana y L. Pardo. “Ferroelectricity of lanthanum modified lead titanate thin films obtained by a diol based sol-gel method". Appl. Phys. A. 68 (1999) 583-592.

6. N.J. Phillips, M.L. Calzada y S.J. Milne. "Sol-Gel derived lead titanate films". J. Non-Crystalline Solids 147\&148, 285-290 (1992).

7. R. Sirera y M.L. Calzada. "Multicomponent solution for the deposition of lead titanate films" Mat. Res. Bull. 30:1, 11-18 (1995)

8. B.A. Tuttle, T.J. Headley, B.C. Bunker, R.W. Schwartz, T.J. Zender, C.L. Hernandez, D.C. Goodnow, R.J. Tissot y J. Michael. "Microstructural evolution of $\mathrm{Pb}(\mathrm{Zr}, \mathrm{Ti}) \mathrm{O}_{3}$ thin films prepared by hybrid metallo-organic decomposition". J. Mater. Res. 10:8 2042-2051 (1995).

9. M. Algueró, M.L. Calzada, C. Quintana y L. Pardo. “Nucleación y crecimiento de la fase perovskita en láminas delgadas de $\mathrm{Pb}_{0.88} \mathrm{La}_{0.08} \mathrm{TiO}_{3}$ ". Bol. Soc Esp. Ceram. Vidrio 37:2-3 113-116 (1998).

10. H. Maiwa y N. Ichinose. “Dielectric properties of $(\mathrm{Pb}, \mathrm{La}) \mathrm{TiO}_{3}$ by multiplecathode sputtering and its application to dynamic random access memory capacitors". Jpn. J. Appl. Phys. 35 4976-4979 (1996).

TABLA I. ESPESOR, $\mathrm{t}$, DISTORSIÓN TETRAGONAL, c/a, RELACIÓN ATÓMICA MEDIA, Pb/Ti, POLARIZACIÓN CONMUTADA CON 300 kV cm ${ }^{-1}$, P ${ }_{\text {SW' }}$ PERMITIVIDAD DIELÉCTRICA A $1 \mathrm{kHz}, \varepsilon^{\prime}$, TANGENTE DE PÉRDIDAS DIELÉCTRICAS A $1 \mathrm{kHz}$, tan $\delta$, Y TEMPERATURA DE LA TRANSICIÓN FERRO-PARAELÉCTRICA, Tt DE LAS LÁMINAS DE PTL20.

\begin{tabular}{|c|c|c|c|c|c|c|c|c|}
\hline $\begin{array}{l}\text { velocidad de } \\
\text { calentamiento }\end{array}$ & $\begin{array}{c}\text { exceso } \\
\mathrm{PbO}\end{array}$ & $\begin{array}{c}t \\
(\mathrm{~nm})\end{array}$ & $c / a$ & $\mathrm{~Pb} / \mathrm{Ti}$ & $\begin{array}{c}\mathbf{P}_{\mathrm{sw}} \\
\left(\mu \mathrm{C} \mathrm{cm}^{-2}\right)\end{array}$ & $\varepsilon^{\prime}$ & $\tan \delta$ & $\begin{array}{c}\mathrm{T}_{\mathrm{t}} \\
\left({ }^{\circ} \mathrm{C}\right)\end{array}$ \\
\hline \multirow[t]{3}{*}{$10^{\circ} \mathrm{C} \min ^{-1}$} & $0 \%$ & 340 & 1.011 & 0.51 & 3.7 & 150 & 0.4 & 233 \\
\hline & $10 \%$ & 350 & 1.004 & 0.63 & 7.4 & 220 & 0.3 & 236 \\
\hline & $20 \%$ & 430 & 1.010 & 0.71 & 5.8 & 300 & 0.6 & 247 \\
\hline \multirow{3}{*}{$\begin{array}{l}\text { calentamiento } \\
\text { rápido }\end{array}$} & $0 \%$ & 380 & 1.004 & 0.57 & 0.6 & 350 & 0.25 & - \\
\hline & $10 \%$ & 330 & 1.004 & 0.63 & 2.8 & 900 & 0.15 & 195 \\
\hline & $20 \%$ & 370 & 1.006 & 0.72 & 4.4 & 700 & 0.12 & 230 \\
\hline
\end{tabular}

